\title{
A Review to the Stability of Discrete Time State- Space Filters using Saturation Non-Linearity
}

\author{
Shiva Jaiswal ${ }^{1}$, Preeti Verma ${ }^{2}$ \\ Assistant Professor, Department of Electronics and Communication Engineering, SRMS CET\&R, Bareilly, India ${ }^{1,2}$
}

\begin{abstract}
The problem concerning the elimination of overflow oscillation in fixed-point state-space digital filter employing saturation arithmetic is considered by various researchers. In this paper a review is done to the finite procedure proposed by T. Oba [1] to test the stability of digital filters under saturation arithmetic.
\end{abstract}

Keywords: Digital filters, finite wordlength, asymptotic stability, nonlinear system.

\section{INTRODUCTION}

When a digital filter is implemented on a digital computer or on special-purpose digital hardware, the filter coefficients are stored in binary registers. These registers can accommodate only a finite number of bits and hence the filter coefficients have to be truncated or rounded-off in order to fit into these register. The finite-word length in recursive digital filter produces non-linearities, namely quantization and overflow. The presence of such non-linearities may result in the instability of the designed system. When dealing with the design and implementation of fixed-point statespace digital filters, it is, therefore, essential to know the conditions under which the filter will be globally asymptotically stable.

\section{SYSTEM DESCRIPTION}

The system under consideration is described by

$$
\begin{aligned}
& x(r+1)=f(y(r))= \\
& {\left[\begin{array}{l}
f_{1}\left(y_{1}(r)\right) \quad f_{2}\left(y_{2}(r)\right) \ldots \ldots \ldots \ldots . \ldots f_{n}\left(y_{n}(r)\right)
\end{array}\right]^{T}} \\
& y(r)=\left[\begin{array}{lr}
y_{1}(r) & y_{2}(r) \ldots \ldots \ldots \ldots . . . . . y_{n}(r)
\end{array}\right]^{T}=A x(r)
\end{aligned}
$$

Where $x(r)$ is an $\mathrm{n}$-vector space, $A=\left[a_{i j}\right]$ is the $\mathrm{n} \mathrm{n} \mathrm{n}$ coefficient matrix, and $\mathrm{T}$ denotes transpose. The saturation nonlinearity is given by

$$
f_{i}\left(y_{i}(r)\right)=\left\{\begin{array}{cc}
1 & y_{i}(r)>1 \\
y_{i}(r) & \left|y_{i}(r)\right| \leq 1 \\
-1 & y_{i}(r)<-1
\end{array}\right.
$$

$\mathrm{i}=1,2,3, \ldots \ldots \ldots \ldots . . \mathrm{n}$ are under consideration.

$\mathrm{Eq}(1)$ is used to describe digital filters with symmetric saturation implemented with finite register length under zero external inputs.

\section{THEOREM 1}

The system described in (1) is asymptotically stable if there exists a positive definite matrix P satisfying

$$
(P)_{i, i}-\sum_{j \neq i}\left(w_{|A|}\right)_{j}(|P|)_{i, j}>0 \quad \text { for } \quad \text { all } \quad i \in J_{|A|}^{c} \quad \text { (2a) }
$$

such that $P-A^{T} P A$ is positive definite.

There are some prerequisite which are to be known before stating the algorithm to calculate $w_{|A|}$ for (2a), they are

a) Stability test is to be done on matrix A, where $A \in R^{n x n}$,

b) The order of the matrix $\mathrm{A}$ is $\mathrm{n}$. 
c) The matrix $B=\left|a_{i j}\right|, i, j=1,2 \ldots n$

d) $\quad \mathrm{J}_{0}=\phi ; \mathrm{J}_{\mathrm{k}}$ contains coordinates indices

e) $\quad \mathrm{n}_{0}=0 ; \mathrm{n}_{\mathrm{k}}$ contains the number of indices of $\mathbf{J}_{\mathrm{k}}$

f) $\quad J_{k}^{c}$ contains the complement indices of $\mathrm{J}_{\mathrm{k}}$

g)

\section{ALGORITHM 1}

The following procedure is proposed by ref [1] with $\mathrm{k}=1$, to obtain $J_{B}, n_{B}$ and $w_{B}=R^{n}$

i) Let $J_{k}$ denotes the list of coordinate indices i's satisfying $\left(B w_{k-1}\right)_{i}<1$, and let $n_{k}$ denotes the number of the indices in $J_{k}$

ii) If $J_{k}=J_{k-1}$, or if $n_{k}=n$, then define $J_{B}=J_{k}, n_{B}=n_{k}$, and

$$
w_{B}=\left\{\begin{array}{ccc}
w_{k-1} & \text { if } & n_{B}<n \\
0 & \text { if } & n_{B}=n
\end{array}\right.
$$

and then exit the loop.

iii) Define $w_{k} \in R^{n}$ such that

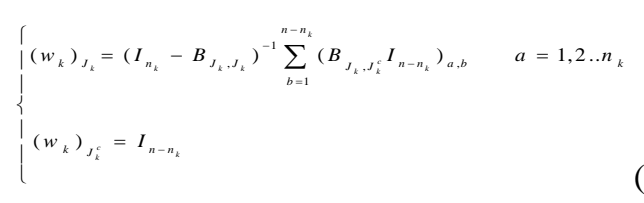

and return to step ( $\mathrm{i}$ ) with $\mathrm{k}=\mathrm{k}+1$.

\section{NUMERICAL EXAMPLE 1}

To illustrate the algorithm for the stability test of fixed-point state-space digital filter with saturation arithmetic, a specific example of a third-order digital filter is considered with

$A=\frac{1}{10}\left[\begin{array}{ccc}1 & 5 & 4 \\ 10 & -2 & 5 \\ 0 & -3 & -1\end{array}\right]$

According to the prerequisite of the algorithm Order of the matrix $\mathrm{A}$ is 3

$$
\begin{aligned}
& B=\frac{1}{10}\left[\begin{array}{ccc}
1 & 5 & 4 \\
10 & 2 & 5 \\
0 & 3 & 1
\end{array}\right] \\
& J_{0}=\phi \\
& n_{0}=0 \text {, and }
\end{aligned}
$$

$$
w_{0}=\left\{\begin{array}{l}
1 \\
1\rceil \\
1 \\
1
\end{array} \mid\right.
$$




\section{Iteration1}

Step (i)

$\left.B w_{0}=\frac{1}{10}\left[\begin{array}{ccc}1 & 5 & 4\rceil\lceil 1\rceil \\ 10 & 2 & 5 \\ 0 & 3 & 1\end{array}\right] \mid \begin{array}{c}\| 1\end{array}\right\rfloor=\left[\begin{array}{c}1 \\ 1.7 \\ 0.4\end{array}\right\rfloor$

$\left(B w_{0}\right)_{i}<1$, where ' $\mathrm{i}$ ' is the indices values satisfying the given condition, in this step it is $\{3\}$ Therefore $\mathrm{J}_{1}=\{3\}$ and $\mathrm{n}_{1}=1$ (number of indexes in $\mathrm{J}_{1}$ )

Step (ii)

$J_{0} \neq J_{1}$ and $n_{1} \neq n$

Step(iii)

$$
\left\{\begin{array}{c}
\left(w_{1}\right)_{J_{1}}=\left(I_{n_{1}}-B_{J_{1}, J_{1}}\right)^{-1} \sum_{b=1}^{3-1}\left(B_{J_{1}, J_{1}^{c}} I_{n-n_{1}}\right)_{a, b} \\
\left(w_{k}\right)_{J_{1}^{c}}=I_{n-n_{1}}
\end{array} \quad a=1\right.
$$

Where $J_{1}^{c}$ contains the complement indexes of $J_{1}$ i.e. $J_{1}^{c}=\{1,2\}$

Now

$$
\begin{aligned}
& \left(w_{1}\right)_{J_{1}}=\left\lceil 1-\frac{1}{10}\right]^{-1} \sum_{b=1}^{2}\left(\left\lceil\begin{array}{ll}
0 & \frac{3}{10}
\end{array}\right]\left[\begin{array}{ll}
1 & 0 \\
0 & 1
\end{array}\right]\right)_{1, b} \\
& \left(w_{1}\right)_{J_{1}}=\left\lceil\frac{9}{10}\right]^{-1} \sum_{b=1}^{2}\left(\left[\begin{array}{ll}
0 & 0.3
\end{array}\right]\right)_{1, b} \\
& \left(w_{1}\right)_{J_{1}}=\left\lceil\frac{10}{9}\right][0.3]=0.3333 \\
& \left(w_{1}\right)_{J_{1}^{c}}=\left[\begin{array}{ll}
1 & 0 \\
0 & 1
\end{array}\right]
\end{aligned}
$$

Now return to step (i) of the algorithm, with $\mathrm{k}=\mathrm{k}+1$ i.e. $\mathrm{k}=2$

\section{Iteration 2}

Step (i)

$w_{1}=\left\{\begin{array}{c}1 \\ 1 \\ 0.3333\end{array}\right]$; since $J_{1}=\{3\}$ therefore

$w_{1}(3)=\left(w_{1}\right)_{J_{1}}$

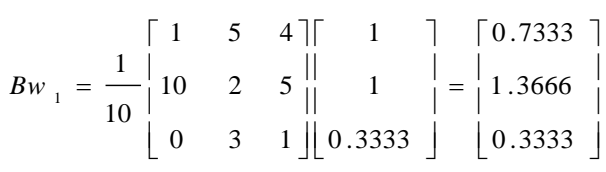

$\left(B w_{1}\right)_{i}<1$, where ' $\mathrm{i}$ ' is the indices values satisfying the given condition, in this step it is $\{1,3\}$

Therefore $J_{2}=\{1,3\}$ and $n_{2}=2$ (the number of indices in $J_{2}$ )

Step (ii)

$J_{1} \neq J_{2}$ and $n_{2} \neq n$

Step (iii)

$\left\{\begin{aligned}\left(w_{2}\right)_{J_{2}}= & \left(I_{n_{2}}-B_{J_{2}, J_{2}}\right)^{-1} \sum_{b=1}^{3-2}\left(B_{J_{2}, J_{2}} I_{n-n_{2}}\right)_{a, b} \quad a=1,2 \\ & \left(w_{2}\right)_{J_{2}^{c}}=I_{n-n_{2}}\end{aligned}\right.$ 
Where $J_{2}^{c}$ contains the complement indexes of $J_{2}$ i.e. $J_{2}^{c}=\{2\}$

Now

$$
\begin{aligned}
& \left(w_{2}\right)_{J_{2}}=\left[\left[\begin{array}{ll}
1 & 0 \\
0 & 1
\end{array}\right]-\frac{1}{10}\left[\begin{array}{cc}
1 & 4 \\
0 & 1
\end{array}\right]\right]^{-1} \sum_{b=1}^{1}\left(\frac{1}{10}\left[\begin{array}{l}
5 \\
3
\end{array}\right][1]\right)_{a, b} \quad a=1,2\left(w_{2}\right)_{J_{2}^{c}}=[1] \\
& \left(w_{2}\right)_{J_{2}}=\left[\begin{array}{cc}
0.9 & -0.4 \\
0 & 0.9
\end{array}\right]^{-1}\left(\begin{array}{l}
0.5 \\
0.3
\end{array}\right)_{a, 1} \quad a=1,2 \\
& \left(w_{2}\right)_{J_{2}}=\left[\begin{array}{l}
0.7037 \\
0.0333
\end{array}\right]
\end{aligned}
$$

Return to step (i) of the algorithm with $\mathrm{k}=\mathrm{k}+1$, i. e $\mathrm{k}=3$

\section{Iteration 3}

Step (i)

$$
\begin{aligned}
& w_{2}=\left[\begin{array}{c}
0.7037 \\
1 \\
0.0333
\end{array}\right] \text {; since } J_{2}=\{1,3\} \text { therefore } \\
& B w_{2}=\frac{1}{10}\left[\begin{array}{ccc}
1 & 5 & 4\rceil[0.7037 \\
10 & 2 & 5 \\
0 & 3 & 1
\end{array}\right][0.0333\rfloor \mid\left[\begin{array}{c}
0.7037 \\
0
\end{array}\right]=\left[\begin{array}{l}
1.0703 \\
0.3333
\end{array}\right]
\end{aligned}
$$

$$
w_{2}\left(\begin{array}{l}
1 \\
3
\end{array}\right)=\left(w_{2}\right)_{J_{2}}
$$

$\left(B w_{2}\right)_{i}<1$, where ' $\mathrm{i}$ ' is the indices values satisfying the given condition, in this step, it is again $\{1,3\}$. Therefore $J_{3}=\{1,3\}$ and $n_{3}=2$ (the number of indices in $J_{3}$ )

Step (ii)

$J_{2}=J_{3}$, and $n_{3} \neq n$

In step (ii) of iteration 3, one of the conditions stated in step (ii) of the algorithm is satisfied. Therefore we will define

$J_{B}=J_{3}=\{1,3\}$

$n_{B}=n_{3}=2$ and

$w_{B}=w_{2}$, since $n_{B}<n$

$\left(w_{B}\right)=(w)_{2}=\left[\begin{array}{c}0.7037 \\ 1 \\ 0.3333\end{array}\right]$

Exit the loop.

To calculate the value of $\mathrm{P}$ for the given A in Numerical Example 1, we will use MATLAB LMI tool box. The matrix $\mathrm{P}$ for given $\mathrm{A}$ in Numerical Example 1 comes out to be

$P=\left[\begin{array}{ccc}0.8951 & -0.0789 & 0.1553 \\ -0.0789 & 0.5423 & -0.0186 \\ 0.1553 & -0.0186 & 1.1414\end{array}\right]$

Following the algorithm stated in IV, for the A given in numerical example 1 we have $J_{|A|}=\{1,3\}$ and $J_{|A|}^{c}=\{2\}$.

Considering $J_{|A|}, J_{|A|}^{c}$ and P, for the given A in Numerical example 1, we will check whether Theorem 1 is satisfied, i.e.

$$
(P)_{i, i}-\sum_{j \neq i}\left(w_{|A|}\right)_{j}(|P|)_{i, j}>0 \quad \text { for } \quad \text { all } \quad i \in J_{|A|}^{c} \quad(3 a)
$$

In our case

$$
(P)_{i, i}-\sum_{j \neq i}\left(w_{|A|}\right)_{j}(|P|)_{i, j}>0 \quad \text { for } \quad \text { all } \quad i \in J_{|A|}^{c}=\{2\} \quad(3 b)
$$




$$
(P)_{2,2}-\left(w_{|A|}\right)_{1}(|P|)_{2,1}-\left(w_{|A|}\right)_{3}(|P|)_{2,3}
$$

$$
(0.5423)-(0.7037) *(|-0.0789|)-(0.3333) *(|-0.0186|)=0.4806
$$

Thus the value of (3c) comes out to be greater than zero. Hence the system considered in the numerical example 1 is judged to be asymptotically stable according to Theorem 1 . The same can also be verified by plotting the state trajectories of the numerical example 1. The figure1 shows that the system under consideration is stable, as the next state of the system reaches zero with increasing iterations i.e. the output reaches zero with zero input

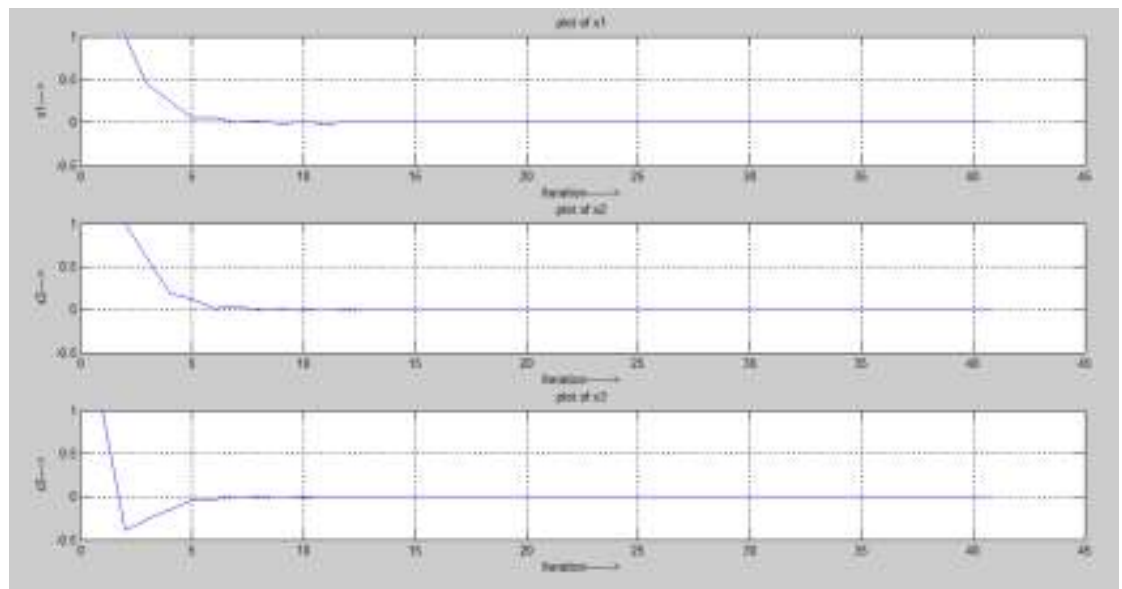

Figure1 Dynamical behavior of the system considered in numerical example

\section{CONCLUSION}

The criteria for the global asymptotic stability of fixed-point state-space digital filters with saturation nonlinearity have been given by several researchers. A finite procedure proposed by Ooba.T [1] ascertains the global asymptotic stability of the system considered in the numerical example. Modification is done to the algorithm proposed by [1] , which is reasonably required and it broaden the scope of stability test from those of earlier results.

\section{REFERENCES}

[1] T. Ooba, Stability of discrete -time systems joined with a saturation operator on state-space, IEEE Trans. Automat. Control 55 (9) (2010) 21532155

[2] D. Liu, A.N. Michel, Asymptotic stability of discrete-time systems with saturation nonlinearities with applications to digital filters, IEEE Trans. Circuits Syst. I 39 (10) (1992) 789-807.

[3] V. Singh, Modified form of Liu-Michel's criterion for global asymptotic stability of fixed-point state-space digital filters using saturation arithmetic, IEEE Trans. Circuits Syst. II 53 (12) (2006) 1423-1425.

[4] H. Kar, An improved version of modified Liu-Michel's criterion for global asymptotic stability of fixed-point state-space digital filters using saturation arithmetic, Digital Signal Process. 20 (4) (2010) 977-981

[5] V.Singh, Elimination of overflow oscillations in fixed-point state-space digital filters using saturation arithmetic, IEEE Trans. Circuits Syst. 37 (6) (1990) 814-818.

[6] V. Singh, A new frequency-domain criterion for elimination of limit cycles in fixed-point state-space digital filters using saturation arithmetic, Chaos, Solutions \& Fractals 34 (3) (2007) 813-816.

[6] H. Kar, V. Singh, A new criterion for the overflow stability of second-order digital filters using saturation arithmetic, IEEE Trans. Circuits Syst. I 45 (3) (1998) 311-313.

[8] V. Singh, A new reliability condition for limit cycle free state-space digital filter employing saturation arithmetic, IEEE Trans. Circuits Syst. 32 (10) (1985) 1070-1071.

[9] H. Kar, V. Singh, Elimination of overflow oscillations in fixed-point state-space digital filters with saturation arithmetic: An LMI approach, IEEE Trans Circuits Syst. II 51 (1) (2004) 40-42.

[10] H. Kar, An LMI based criterion for the nonexistence of overflow oscillations in fixed-point state-space digital filters using saturation arithmetic, Digital Signal Process. 17 (3) (2007) 685-689.

[12] T. Ooba, Stability of linear discrete dynamics employing state saturation arithmetic, IEEE Trans. Automat. Control 48 (4) (2003) 626-630 